\title{
Workshop HPC und E-Health
}

\author{
Das Interesse an der E-Health-Strategie der FMH und dem Projekt Health Pro- \\ fessional Card HPC ist gross: Über dreissig Gesellschaften sind einer Einladung \\ zu einem Workshop gefolgt und haben am 3. September 2008 ihre Delegier- \\ ten nach Bern gesandt - um sich von der Projektgruppe informieren zu lassen, \\ aber auch um Antworten auf offene Fragen zu erhalten.
}

Dr. med. Max Giger, Mitglied des Zentralvorstandes $\operatorname{der} \mathrm{FMH}$
Initiiert hat den Workshop Dr. med. Monique Gauthey, die im FMH-Zentralvorstand neu für das Ressort eHealth verantwortlich ist und die Veranstaltung mit einem Referat zu den europäischen und schweizerischen Entwicklungen im Bereich E-Health und HPC eröffnet (siehe Editorial dieser Ausgabe).

Im ersten Teil des Workshops zeigt Zentralvorstandsmitglied Dr. Max Giger Nutzen und Einsatzmöglichkeiten der HPC auf. Dr. Georg Kassowitz und Dr. Harald Heuser orientieren anschliessend über die operative und technische
Umsetzung. Im zweiten Teil des Workshops thematisiert die E-Health-Verantwortliche der FHM, Dr. Judith Wagner, die für die FMH relevanten Entwicklungen im Bereich E-Health.

Nach dem Workshop ist klar: HPC und E-Health werden den ärztlichen Alltag in $\mathrm{Zu}-$ kunft stark prägen. Umso wichtiger ist es, dass die Ärzteschaft aktuell informiert ist. Deshalb haben die Referentinnen und Referenten des Workshops ihre Vorträge für die Leserschaft der SÄZ zusammengefasst - Sie finden ihre Beiträge auf den folgenden Seiten.

\section{Nutzen und Einsatz der HPC}

Die Health Professional Card (HPC) dient allen Ärztinnen und Ärzten, die in der Schweiz arbeiten, als Ausweis und ermöglicht eine physische und virtuelle bzw. elektronische Authentisierung. Mit der HPC lassen sich Dokumente elektronisch unterzeichnen und gesichert transportieren bzw. empfangen. Dank der generell offenen Struktur kann sie für nationale und internationale E-Health-Anwendungen im medizinischen Kontext verwendet werden. Die HPC gewährleistet Interoperabilität, d.h. sie ermöglicht Ärztinnen und Ärzten sowie bei Bedarf weiteren medizinischen Berufsgruppen den elektronischen Austausch von Informationen. Die Empfänger können die Informationen so benutzen, wie wenn sie diese selbst generiert hätten. Beispielsweise lassen sich Vitaldaten, Laborwerte und Berichte von Zuweisenden direkt in die eigenen elektronischen Krankenakten übertragen. Die HPC ist kompatibel mit der Versichertenkarte, die voraussichtlich per 1. Januar 2010 durch die Krankenversicherer ausgehändigt wird.

Durch die HPC positioniert sich die FMH als wichtige Partnerin im Bereich E-Health. Voraus- setzung dafür waren die zukunftsweisenden strategischen Entscheide durch die Ärztekammern von Dezember 2005 und 2006. Nach einer sich an Vorgaben der WTO anlehnenden Ausschreibung starteten im August 2007 die Projektarbeiten mit Swisscom IT Services. Die Arbeiten an den Pilotapplikationen wurden im Juli 2008 aufgenommen. 2009 erfolgt die Auslieferung der HPC an die Mitglieder der FMH und andere Ärzte. Sämtliche Mitglieder der FMH erhalten dann auch die notwendigen Unterlagen zur Registrierung.

\section{Beispiele von Nutzungsmöglichkeiten}

Die HPC ermöglicht den sicheren elektronischen Verkehr mit myFMH, u.a. die Mutation von eigenen Personendaten. Den Fachgesellschaften bzw. ihren Mitgliedern werden die notwendigen Informatikstrukturen zur Verfügung gestellt, damit diese den Besuch anerkannter Fortbildungsveranstaltungen mit der HPC registrieren können. Damit vereinfachen sich der Nachweis von Fortbildungscredits und die darauf basierende Aushändigung des Fortbildungszertifikats. Die 
Ärztinnen und Ärzte in Weiterbildung werden die Einträge ins Logbuch elektronisch gesichert vornehmen können. Und die Leiterinnen und Leiter der Weiterbildungsstätten können Zeugnisse elektronisch unterzeichnen. Um die wichtigsten Praxissoftwareprogramme an die HPC anzubinden, wird ausserdem der Einsatz der HPC in Kooperation mit der Swiss Medical Suite geprüft. Last but not least: Der Einsatz von Entscheidungshilfen (Decision Support Systems) im
Rahmen der elektronischen Verordnung von Arzneimitteln wird eine Erhöhung der Medikationssicherheit ermöglichen.

Diese Nutzungsmöglichkeiten der HPC zeigen den Weg auf, welcher zum Ziel führt: gesicherte und effiziente dem Patientenpfad folgende elektronische Kommunikation und Dokumentation. Dadurch werden Ärztinnen und Ärzte Zeit für sich und ihre Patientinnen und Patienten gewinnen.

\section{Dr. Harald Heuser, Mitglied des Projektteams HPC-FMH}

\section{Datensicherheit und Technik der FMH-HPC}

\section{Einsatzgebiete und Technologie}

Mit der HPC (Health Professional Card) stellt die FMH auf der Basis von internationalen Standards eine Kernkomponente für die drei folgenden Grundanwendungen bereit:

- das Erstellen von digitalen Signaturen;

- die Authentisierung von Personen an elektronischen Systemen;

- die Verschlüsselung von Daten bei der Übermittlung oder Archivierung.

Beim Einsatz der HPC gelangen die Kryptotechniken nach dem Private-Public-Key-Verfahren zur Anwendung. Dieses Verfahren ist international verbreitet und wird von zahlreichen gängigen Standard-Softwareapplikationen ebenfalls unterstützt. In einer sicheren Umgebung wird dazu ein Krypto-Schlüsselpaar erzeugt, bestehend aus einem privaten und einem öffentlichen Schlüssel. Der private befindet sich ausschliesslich auf der HPC, der öffentliche (public) wird als Zertifikat in einem Web-Service veröffentlicht und gepflegt.

\section{Voraussetzung für die Nutzung}

Voraussetzung für die Nutzung der FMH-HPC ist ein handelsüblicher $\mathrm{PC}$ mit den gängigen Betriebssystemen Windows, Mac oder Linux, ein Standard-Kartenlesegerät, eine FMH-HPCTreibersoftware und eine Applikation, mit der die oben genannten Grundanwendungen durchgeführt werden können. Beispiele für solche Applikationen sind Outlook und Acrobat Professional.

Für den Einsatz der HPC in Verbindung mit spezifischen Applikationen wie Praxismanagementsystemen steht ein Software Development Kit (SDK) zur Verfügung, so dass der betreffenden Hersteller die Nutzung der HPC sehr schnell in seine spezifische Applikation integrieren kann.

\section{Sicherheit}

Die HPC der FMH gibt es in mehreren Zertifikatsversionen, die sich durch verschiedene Sicherheitsstufen mit unterschiedlichen Rechtswirkungen unterscheiden. Je nach Wunsch wird das entsprechende Zertifikat oder auch mehrere auf der HPC aufgebracht. Die höchste Sicherheitsstufe, mit dem entsprechenden Zertifikat, erlaubt gar das Erstellen elektronischer Signaturen, die nach schweizerischem Gesetz den handschriftlichen Unterschriften gleichgestellt sind. Möglich macht dies die Zertifizierung, über die Swisscom als Lieferant der HPC verfügt. Als eines von nur vier anerkannten Unternehmen in der Schweiz erfüllt sie die hohen Sicherheitsanforderungen des Schweizer Signaturgesetzes.

\section{Erstellung und Prüfung elektronischer Signaturen}

Die elektronische Signatur wird mit der FMHHPC in zwei Schritten erzeugt: Zuerst wird ein Hash-Wert der betreffenden Datei oder Nachricht gebildet. Der Hash-Wert einer Datei bzw. einer Nachricht ist vergleichbar mit dem Fingerabdruck einer Person. Dann wird der Hash-Wert mit dem privaten Schlüssel des Signierenden verschlüsselt und der Datei oder der Nachricht angehängt. Das öffentliche Zertifikat des Signierenden wird ebenfalls angehängt.

Durch Überprüfung des Hash-Werts kann nun nachträglich zweifelsfrei festgestellt werden, ob die Datei oder die Nachricht noch unverändert ist oder ob sie verändert wurde. Durch Überprüfen des öffentlichen Zertifikats kann zweifelsfrei die Identität des Signierenden und die Gültigkeit der Signatur festgestellt werden. Die Gültigkeit einer Signatur kann verlängert werden, indem die signierte Datei oder Nachricht erneut mit einem Zertifikat signiert wird, das eine längere Gültigkeitsdauer hat. 


\section{Verschlüsselung und Entschlüsselung}

Die zu verschlüsselnde Datei oder Nachricht wird mit dem öffentlichen Zertifikat des gewünschten Empfängers verschlüsselt und anschliessend dem Empfänger über eine beliebige Datenverbindung zugestellt. Die verschlüsselte Datei oder Nachricht kann nur vom betreffenden Empfänger mit seinem privaten Zertifikat wieder lesbar gemacht werden. Damit ist sichergestellt, dass die betreffende Datei oder Nachricht vom dem Ort und ab dem Zeitpunkt, an dem der Absender die betreffende Datei oder Nachricht verschlüsselt, bis zu dem Ort und zu dem Zeitpunkt, an dem der Empfänger die betreffende Datei oder Nachricht entschlüsselt, gegenüber jedem Dritten vertraulich ist.

Eine verschlüsselte Datei oder Nachricht ist vergleichbar mit einem versiegelten Brief, der von einem Boten an einen Empfänger übergeben wird, nachdem sich der Empfänger mit seinem Ausweis identifiziert hat. Eine unverschlüsselte Datei oder Nachricht ist vergleichbar mit einer Postkarte im Postverkehr.

\section{Ausblick}

Mit der HPC der FMH werden Grundanwendungen für den flexiblen und trotzdem sicheren Informationsaustausch im Gesundheitswesen ermöglicht. Die HPC hat damit eine Katalysatorwirkung für die Realisierung darauf aufbauender Applikationen, mit denen vielfältige Arbeitserleichterungen im Alltag des Gesundheitswesens erreicht werden können. Die FMH unterstützt solche weiterführenden Applikationen und wird dazu im Rahmen der HPC-Pilotphase weitere Schritte unternehmen.

\section{Das Projekt FMH-HPC (Health Professional Card)}

\section{Dr. Georg Kassowitz,} Projektleiter HPC-FMH
Die Einführung der FMH-HPC, einer Karte in Kreditkartengrösse für die Health Professionals, soll den Trägerinnen und Trägern sinnvolle Instrumente im Alltag näherbringen und die Möglichkeiten moderner elektronischer Hilfsmittel erschliessen. Weil ein solch komplexes Vorhaben gut koordiniert und abgestimmt sein muss, wurde das Projekt FMH-HPC ins Leben gerufen.

\section{Abbildung 1}

Projektablauf mit einzelnen Phasen.

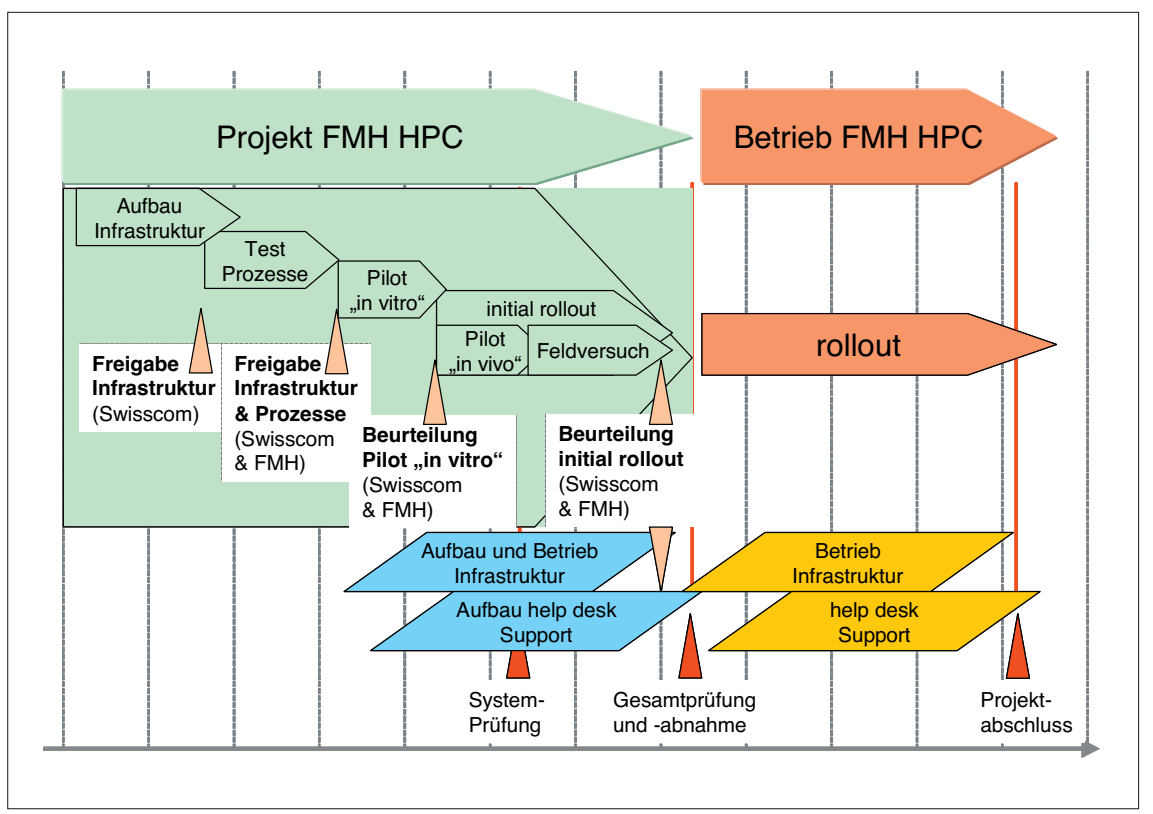

Das Projekt ist in mehrere Phasen gegliedert und wird in Absprache mit dem Lieferanten (Swisscom IT Services) koordiniert. Nach dem Aufbau der benötigten Infrastruktur werden in Pilotphasen die ersten Praxis-Tests der FMH HPCs bei einem begrenzten Kreis von Anwendern durchgeführt. Obwohl es sich bei den eingesetzten Hilfsmitteln um bekannte und bewährte Technologien handelt, könnten im Zusammenspiel der Komponenten Probleme auftauchen. Wenn dies geschieht, soll die Behebung rasch und sicher erfolgen können. In einer zweiten Pilotphase werden Karten an eine Stichprobe von Nutzenden ausgegeben, welche die kompletten Anforderungen möglichst gut repräsentiert. Das Testen, Ausprobieren und Sammeln von Erfahrungen soll die Behebung von allfällig auftauchenden Mängeln oder Problemen erlauben.

Erst nach erfolgreicher Beendigung der praxisnahen Pilotphasen kann die Auslieferung der HPC an Professionals gestartet werden. Die sogenannte Registrierung, dank welcher man als Berechtigter eine FMH-HPC erhalten kann, wird einen wesentlichen Teil der Verbreitung ausmachen. Wer als Professional den erforderlichen Antrag ausfüllt und die notwendigen Unterlagen zustellt, erhält innerhalb weniger Wochen oder gar Tagen den neuen Ausweis per Post zugestellt. Da die Herausgabe des Ausweises nicht durch die FMH selbst geschieht, sondern durch eine be- 
rechtigte und streng überwachte Stelle erfolgen muss, werden hier hohe Sicherheitsanforderungen an die Produktion zu erfüllen sein.

Für den Besitzer einer FMH-HPC wird es möglich sein, mit geringen Aufwendungen bezüglich Infrastruktur oder sogar mit der bestehenden Ausrüstung seine Karte in einer sogenannten offenen Informatikumgebung sicher zu nutzen.

\section{E-Health - FMH}

Dr. Judith Wagner, Leiterin eHealth FMH

Im Juni 2007 hat der Bundesrat die nationale E-Health-Strategie verabschiedet, die vom BAG zusammen mit den Kantonen erstellt wurde. Die Umsetzung dieser Strategie soll über das Anfang Jahr gegründete und von Bund und Kantonen gemeinsam getragene Koordinationsorgan eHealth erfolgen. Dieses Koordinationsorgan erarbeitet Empfehlungen zuhanden aller Akteure im Gesundheitswesen. Es besteht ein politischer Wille, die Empfehlungen je nach Bedarf rechtlich zu verankern [1].

Das Koordinationsorgan besteht aus einer Geschäftsstelle mit drei Mitarbeitern und zahlreichen Arbeitsgruppen. Es wird geleitet von einem Steuerungsausschuss mit Vertretern der Kantone und des Bundes, unter Vorsitz von Bundespräsident Pascal Couchepin. Eine beratende Begleitgruppe mit verschiedensten Interessenvertretern wird vor wichtigen Beschlüssen angehört. Die fachlich-inhaltliche Arbeit erfolgt in verschiedenen Teilprojekten, die zum Teil wiederum mit Untergruppen arbeiten. Die Arbeit der Teilprojekte wird im Projektleitungsgremium koordiniert. Der Zeitplan ist sehr ambitiös, eine Vernehmlassung erster Lösungsvorschläge soll schon im November erfolgen.

Die nationale E-Health-Strategie wurde ohne Einbezug der Ärzteschaft erstellt, obwohl diese neben den Patienten die Hauptbetroffenen sind. Umso wichtiger ist es, dass sich die Ärzteschaft nun über ihre Positionen auf dem Gebiet E-Health klar wird - und diese auch konsequent einnehmen kann.

Die FMH ist überzeugt, dass E-Health-Projekte ohne Einbezug der Ärzteschaft nicht erfolgreich sein können, und vertritt die Ärzteschaft in den verschiedenen Gremien entsprechend.

Sie insistiert darauf, dass die Erwartungen an E-Health realistisch sein und bleiben müssen
Offen heisst in diesem Zusammenhang, dass sich Produkte ohne Rücksicht auf Marke oder Herkunft frei gewählt und kompatibel einsetzen lassen. Der Datenschutz ist dabei dank technologischer Unterstützung selbstverständlich umfassend gewährleistet und der reibungslose Betrieb sichergestellt.

und dass die ICT nicht dazu verwendet werden darf, Verhaltensänderungen herbeizuführen, d.h. Änderungen von Arbeitsweisen und Abläufen zu erzwingen ( do not use a computer to force a change of behaviour»).

So vertritt die FMH beispielsweise folgende Positionen: Mit E-Health-Tools sollen die Voraussetzungen für einen elektronischen Austausch geschaffen werden. Es darf nicht versucht werden, die Arbeitsweise der Ärzte zu standardisieren, sondern Austauschprozesse müssen harmonisiert werden. Der Fokus muss auf dem Austausch von Daten zwischen verschiedenen Behandelnden liegen. Beispielsweise müssen nicht Austrittsberichte inhaltlich komplett standardisiert werden, um sie austauschen zu können. Hingegen müssen sich Absender, Empfänger und die betroffenen Patienten eindeutig identifizieren lassen.

Aus Sicht der Ärzteschaft ist es besonders wichtig, dass die Verwendung von E-HealthTools freiwillig bleibt. Ebenso wichtig ist es, dass für Patienten und Ärzte ein umfassender Datenschutz sichergestellt ist, denn Vertrauen wird auch in Zukunft und mit E-Health grundlegend für den Behandlungsprozess bleiben.

Die FMH schlägt die Gründung einer Arbeitsgruppe E-Health vor, in welcher Positionen im Bereich E-Health abgestimmt und Stellungnahmen koordiniert werden können, insbesondere auch bezüglich des Koordinationsorgans eHealth. Wesentlich ist aber auch die generelle Positionierung der Ärzteschaft bezüglich E-Health. Deshalb sollen in dieser Arbeitsgruppe auch Inhalte einer E-Health-Strategie der FMH thematisiert werden.

1 Vgl. hierzu: www.bag.admin.ch/themen/ krankenversicherung/04108/index.html?lang=de 


\section{Health Professional Card (HPC) - Questions and Answers}

Wer kann alles eine HPC beziehen? Welche Voraussetzungen müssen erfüllt werden?

Alle Ärzte, die in der Schweiz tätig sind, können eine HPC beziehen. Mitglieder der FMH erhalten den HPC-Antrag automatisch zugestellt. Für Ärzte, die nicht Mitglied bei der FMH sind, ist ein Nachweis der Qualifikation erforderlich. Darüber hinaus können noch folgende Personen eine HPC beziehen:

- Mitarbeiter eines Arztes gemäss den Angaben des Arztes und

- Mitarbeiter einer Gesundheitseinrichtung (z.B. Spital) gemäss den Angaben der Leitung der betreffenden Gesundheitseinrichtung.

Wie hoch sind die Kosten für eine HPC? Und wie hoch sind die Folgekosten?

Die Karte mit dem einfachen Zertifikat zum Verkehr mit myfmh ist im FMH-Mitgliederbeitrag eingeschlossen. Die Zertifikate für eine rechtsgültige Unterschrift können dank der Vermittlung über die FMH zu Tiefstpreisen von 20 bis 50 Franken bestellt werden. Die marktüblichen Preise für qualifizierte Zertifikate sind im Vergleich fünf- bis achtmal teurer. Die Folgekosten sind abhängig von der Anzahl Zertifikate, welche die Ärztin benötigt.

Welches sind die technischen Voraussetzungen, damit ich die HPC in der Praxis benutzen kann?

Für die Benutzung einer HPC sind ein StandardKartenlesegerät, eine HPC-Treiber-Software und eine Applikation, die mit Standardzertifikaten arbeitet (z.B. Outlook, Acrobat Professional usw.), erforderlich.

Standard-Kartenlesegeräte sind teilweise bereits in Laptops und PCs eingebaut oder können optional zusammen mit der HPC erworben werden.

Die HPC-Treibersoftware wird zum Download oder optional auf CD verfügbar gemacht werden und folgende Plattformen unterstützen:

- Windows 2000, XP und Vista

- Mac OS X für Intel / Power PC

- Mindestens eines der folgenden Linux-Derivate: Fedora/Red Hat, Ubuntu, Suse.

Für die Nutzung mit der Versichertenkarte wird optional ein Zertifikat verfügbar sein, das den Zugriff auf die Versichertenkarte ermöglicht.
Wie sicher ist die HPC?

Die HPC verfügt über die derzeit höchste Sicherheitsstufe. Die Kryptochips der HPC und die darauf verwendeten elektronischen Schlüssel entsprechen den gesetzlichen Vorgaben für rechtsgültige elektronische Signaturen gemäss dem Schweizer Signaturgesetz und werden nach einem gesetzlich anerkannten Verfahren einer eindeutig identifizierten Person zugeordnet. Die gesetzlichen Voraussetzungen zur Herausgabe einer solchen HPC können zurzeit nur von vier zertifizierten Unternehmen erfüllt werden. Elektronische Daten lassen sich darüber hinaus so verschlüsseln, dass nur der Empfänger bzw. die berechtigte Person sie lesen kann.

Ist die Interoperabilität der FHM-HPC mit HPCs in anderen Ländern und für andere Berufsgruppen gewährleistet?

Die HPC basiert auf internationalen Standards. Damit ist gewährleistet, dass die HPC weltweit mit allen anderen Signaturkarten interoperabel ist, die ebenfalls auf diesen internationalen Standards basieren.

Kann ich mit der HPC Daten gesichert zu einem HIN-Teilnehmer übertragen und umgekehrt?

Vortests haben ergeben, dass ein HIN-Teilnehmer eine digital signierte E-Mail an einen beliebigen Teilnehmer verschicken kann. Umgekehrt funktioniert dies noch nicht. Die notwendigen Anpassungen am ASAS-Client zur Herstellung der Interoperabilität mit der HPC wurden von HIN zugesichert.

Was bietet die HPC mehr gegenüber den HINDiensten?

Mit der HPC lässt sich der Teilnehmerkreis für sicheren Datenaustausch erweitern. Der gesicherte Datenaustausch ist weltweit möglich und nicht auf Teilnehmer beschränkt, die ein HIN-Abo besitzen.

Mit der HPC lässt sich der sichere Datenaustausch vereinfachen und auf der Basis kostenloser Standardapplikationen realisieren. Zudem können Zugänge zu Datendiensten realisiert werden, die nicht über ein HIN-Abo erreichbar sind.

Die HPC erlaubt es, Daten digital zu signieren. Der Empfänger kann zweifelsfrei feststellen, 
wer die Daten signiert hat und ob die Daten auf dem Übertragungsweg verändert wurden.

Kann ich mich mit der HPC in einem Spital einloggen?

Wenn das Spitalsystem das HPC-Zertifikat erkennt und kommunizieren will, ist ein Login auch in einem Spital möglich.

Kann ich mit der HPC Dokumente elektronisch signieren, so dass diese elektronische Signatur einer handschriftlichen Signatur vor dem Gesetz gleichgestellt ist?

Sie können mit der HPC Dokumente elektronisch signieren. Wenn dazu auf der HPC ein qualifiziertes Zertifikat vorhanden ist und verwendet wird, ist diese Signatur vor dem Gesetz der handschriftlichen Signatur gleichgestellt.

Was unterscheidet die Zertifikate der HPC von Zertifikaten, die kostenlos im Internet beschafft werden können?

Die im Internet erhältlichen Zertifikate sind in der Regel technisch ebenso Standardzertifikate wie die Zertifikate der HPC. Sie sind in der Regel ebenfalls interoperabel.

Unterschiedlich sind die Prozesse zur Registrierung der Zertifikatsinhaber, die Zuordnung der Zertifikatsinhaber zu den Kryptoschlüsseln und die Sicherheitsvorkehrungen zur Herstellung und Aufbewahrung der Kryptoschlüssel.

Für die HPC wurde ein Hersteller gewählt, der die höchsten gesetzlichen Sicherheitsanforderungen erfüllt.

Kann ich die HPC zusammen mit einem HIN-Abo verwenden?

Die HPC kann grundsätzlich an jedem PC eingesetzt werden, unabhängig vom Internetanschluss, solange der Internetanschluss die Daten transparent weiterleitet. Das ist derzeit beim HIN-Abo nicht gegeben. Die notwendigen Modifikationen zur Interoperabilität mit der HPC wurden von HIN zugesichert.

Warum machen wir eine Karte und keinen USBStick - ist doch viel komplizierter?

Karte und USB-Stick können beide grundsätzlich als elektronische HPC verwendet werden. Der Sichtausweis-Charakter der HPC kann nur mit der Karte realisiert werden. Ausserdem ist der Krypto-Stick derzeit noch um ein Vielfaches teurer als die Krypto-Karte. Deshalb hat sich die FMH für eine Karte entschieden. Ein geeigneter USB-Stick wird zu gegebener Zeit optional verfügbar sein. 